\title{
Optimization-based subdivision algorithm for reachable sets
}

\author{
Wolfgang Riedl *
}

May 2014

\begin{abstract}
This paper shows how an optimization-based approach to calculate reachable sets can be improved by using a subdivision-algorithm.
\end{abstract}

\section{Optimization-based algorithm for reachable sets}

The reachable set $R$ (sometimes also called attainable set) at a given time $T$ of a nonlinear control system

$$
\dot{x}(t)=f(t, x(t), u(t)), x\left(t_{0}\right)=x_{0}, u(t) \in U, t \in\left[t_{0}, T\right]
$$

is the union of the endpoints of all feasible solutions.

This set can be approximated using an optimization-based approach (e.g. [1]). The basic idea behind this algorithm is, that we choose an initial bounding box $B=\left[a_{1}, b_{1}\right] \times\left[a_{2}, b_{2}\right] \times \ldots$, discretize this box into a grid $G$ (e.g. an eqidistant grid) and solve the optimal control problem (OCP)

$$
\min \|g-x(T)\|^{2} \quad \text { subject to } \dot{x}(t)=f(t, x(t), u(t)), u(t) \in U \text { and } x\left(t_{0}\right)=x_{0}
$$

using direct discretization for every gridpoint $g \in G$. The union of the endpoints of the calculated solutions now approximates the reachable set.

\section{Grid construction via subdivision}

The biggest performance problem of the optimization-based algorithm is, that we have to solve many optimization problems, which can be very expensive. To address this issue we use some ideas from subdivision algorithms (e.g. [3], [4]) to reduce the number of gridpoints and therefore the number of optimization problems.

\section{Definition 1.}

$$
F: \mathbb{R}^{n} \rightarrow \mathbb{R}^{n}, F(g)=x(T ; g) \text { where } x \text { is the solution of the OCP (1). }
$$

With this definition we can show that the reachable set $R$ is the global attractor since $F(R)=$ $R$ and $F(g) \in R$ for every $g \in \mathbb{R}^{n}$.

Algorithm 1. Given: Initial collection $\mathcal{B}_{0}=\{B\}$

${ }^{*}$ Corresponding author: e-mail: wolfgang.riedl@uni-bayreuth.de, Chair of Applied Mathematics, Department of Mathematics, University of Bayreuth, 95440 Bayreuth, Germany 
1. Subdivision: Construct a new collection $\hat{\mathcal{B}}_{k}$ such that

$$
\bigcup_{B \in \hat{\mathcal{B}}_{k}} B=\bigcup_{B \in \mathcal{B}_{k-1}} B
$$

and

$$
\max _{B \in \hat{\mathcal{B}}_{k}} \operatorname{diam}(B)=\theta_{k} \cdot \max _{B \in \mathcal{B}_{k-1}} \operatorname{diam}(B) \text { with } 0<\theta_{\min } \leq \theta \leq \theta_{\max }<1
$$

2. Selection: Define the new collection $\mathcal{B}_{k}$ by

$$
\mathcal{B}_{k}=\left\{\hat{B} \in \hat{\mathcal{B}}_{k}: \exists B \in \mathcal{B}_{k-1}, \exists g \in B \text { such that } F(g) \in \hat{B}\right\}
$$

In the two-dimensional case, we subdivide our initial bounding box into four smaller boxes (i.e. $\theta=0.5$ ) by solving the OCP on an equidistant $3 \times 3$ grid. In the next step we drop all boxes that do not contain at least one endpoint of the nine calculated solutions and we subdivide the remaining boxes in the same way as before. This will be repeated until the grid is dense enough.

Figure 1: First step of the al- Figure 2: Second step of the gorithm.

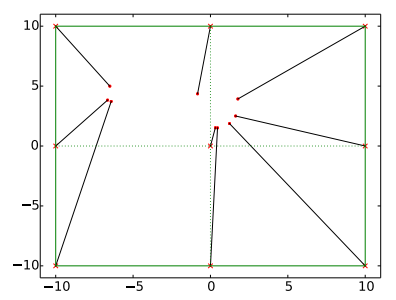
algorithm.

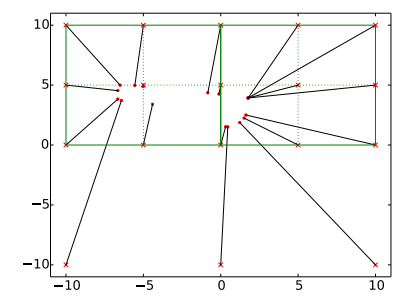

Figure 3: Third step of the algorithm.

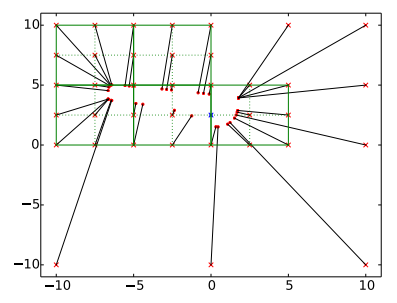

\section{Numerical example}

The Rayleigh-problem (e.g. [2]) with initial bounding box $[-10,10]^{2}$ can be used as an example to illustrate the improvements of the subdivision algorithm:

$$
\begin{aligned}
& \dot{x}_{1}(t)=x_{2}(t), \\
& \dot{x}_{2}(t)=-x_{1}(t)+x_{2}(t) \cdot\left(1.4-0.14 \cdot x_{2}(t)^{2}\right)+4 \cdot u(t),
\end{aligned}
$$

$u(t) \in[-1,1], t \in[0,2.5]$ and $x_{1}(0)=x_{2}(0)=-5$. Figure 4 shows the results using the optimization-based algorithm on a $33 \times 33$ grid and Figure 5 the result of the subdivision with the same density of gridpoints near the set. Table 1 compares the cpu-times to calculate the reachable set using the optimization-based algorithm and the subdivision algorithm and Table 2 shows the number of needed gridpoints for both versions. 
Figure 4: Example using a full grid.

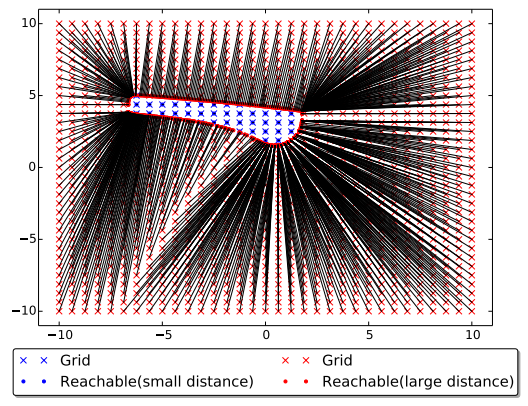

Figure 5: Example using subdivision.

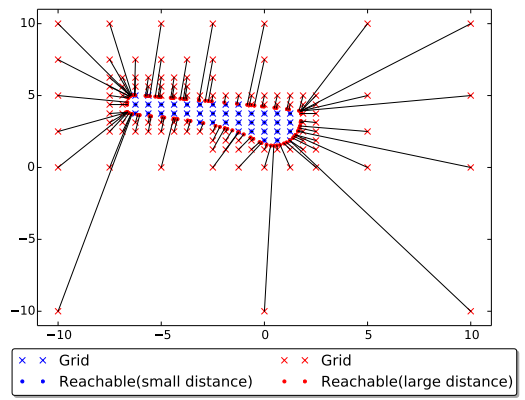

Table 1: CPU-times

\begin{tabular}{rrrr} 
grid & full grid & subdivision & speedu \\
\hline $33 \times 33$ & $1 \mathrm{~m} 27.933 \mathrm{~s}$ & $14.109 \mathrm{~s}$ & 6.2 \\
$49 \times 49$ & $3 \mathrm{~m} 21.253 \mathrm{~s}$ & $25.206 \mathrm{~s}$ & 8.0 \\
$65 \times 65$ & $5 \mathrm{~m} 48.130 \mathrm{~s}$ & $31.794 \mathrm{~s}$ & 10.9 \\
$97 \times 97$ & $13 \mathrm{~m} 05.713 \mathrm{~s}$ & $54.335 \mathrm{~s}$ & 14.5 \\
$129 \times 129$ & $22 \mathrm{~m} 53.810 \mathrm{~s}$ & $1 \mathrm{~m} 06.836 \mathrm{~s}$ & 20.6 \\
$193 \times 193$ & $51 \mathrm{~m} 11.260 \mathrm{~s}$ & $1 \mathrm{~m} 58.871 \mathrm{~s}$ & 25.8 \\
$257 \times 257$ & $1 \mathrm{~h} 30 \mathrm{~m} 49.837 \mathrm{~s}$ & $2 \mathrm{~m} 23.577 \mathrm{~s}$ & 38.0 \\
\hline
\end{tabular}

Table 2: Number of gridpoints

\begin{tabular}{crrr} 
grid & full grid & subdivision & speedup \\
\hline $33 \times 33$ & 1089 & 150 & 7.3 \\
$49 \times 49$ & 2401 & 310 & 7.7 \\
$65 \times 65$ & 4225 & 354 & 11.9 \\
$97 \times 97$ & 9409 & 755 & 12.5 \\
$129 \times 129$ & 16641 & 995 & 16.7 \\
$193 \times 193$ & 37249 & 2353 & 15.8 \\
$257 \times 257$ & 66049 & 3201 & 20.6 \\
\hline
\end{tabular}

\section{References}

[1] R. Baier, M. Gerdts and I. Xausa, Numer. Algebra Control Optim. 3, 519-548 (2013).

[2] R. Baier and M. Gerdts, Proceedings of the European Control Conference (ECC) 2009, Budapest, Hungary, pp.97-102.

[3] M. Dellnitz and A. Hohmann, Numer. Math. 75, 293-317 (1997).

[4] L. Grüne, Asymptotic Behavior of Dynamical and Control Systems under Perturbation and Discretization (Springer-Verlag, Berlin-Heidelberg, 2002), pp. 178-194.

This paper was published on Dec. 22, 2014 in Proceedings in Applied Mathematics and Mechanics, Volume 14, Issue 1, pages 937-938 (DOI: 10.1002/pamm.201410449). 